\title{
Chondrosarcoma masquerading as an Abscess in the Gluteal Region
}

\author{
${ }^{1}$ Misbah Anjum, ${ }^{2}$ Sharmila P Surhonne, ${ }^{3}$ Pannaga P Kumar, ${ }^{4} S$ Swarna Kamala, ${ }^{5}$ Rizni Mansoor
}

\begin{abstract}
Pelvic chondrosarcomas (CHSs) are malignant cartilaginous tumors. Due to their slow growth, CHSs tend to cause symptoms over a prolonged period of time, and their diagnosis might be delayed. Since radiotherapy and chemotherapy have proved ineffective in the treatment of $\mathrm{CHSs}$, wide surgical excision remains the treatment of choice. Although $\mathrm{CHSs}$ rarely metastasize and have a very good prognosis after surgery, local recurrence is a quite common occurrence especially following inadequate excision. In this report, we present a case of pelvic CHS mistaken for an abscess.
\end{abstract}

Keywords: Abscess, Arthritis, Chondrosarcoma, Gluteal swelling.

How to cite this article: Anjum M, Surhonne SP, Kumar PP, Kamala SS, Mansoor R. Chondrosarcoma masquerading as an Abscess in the Gluteal Region. J Med Sci 2018;4(1):20-22.

Source of support: Nil

Conflict of interest: None

\section{INTRODUCTION}

Chondrosarcoma is a rare malignant tumor that produces cartilaginous matrix and accounts for 17 to $24 \%$ of primary malignant bone tumors. The estimated overall incidence of CHSs is 1 in 200,000 per year, and it is the third most frequent malignant bone tumor after multiple myeloma and osteosarcoma. The majority of patients are between 35 and 60 years old with equal sex distribution. ${ }^{1}$ Chondrosarcomas can either arise de novo as primary tumors, or less frequently, they can originate from previously existing benign cartilaginous tumors, such as osteochondromas and enchondromas. ${ }^{2}$

\section{CASE REPORT}

We report the case of a 45-year-old male with complaints of low back ache (nocturnal), radiating to the right leg since 4 months, for which he received around

\footnotetext{
1,3-5 Postgraduate Student, ${ }^{2}$ Professor and Head

${ }^{1-5}$ Department of Pathology, RajaRajeswari Medical College \& Hospital, Bengaluru, Karnataka, India

Corresponding Author: Misbah Anjum, Postgraduate Student Department of Pathology, RajaRajeswari Medical College \& Hospital, Bengaluru, Karnataka, India, Phone: +919535573748 e-mail: dr.misbah.anjum@gmail.com
}

50 gluteal intramuscular injections for pain relief by local practitioners. Following this, he developed a swelling in the right gluteal region and inner aspect of proximal thigh.

Hematological and biochemical parameters were normal, except erythrocyte sedimentation rate (ESR) and alkaline phosphatase (ALP) which were elevated.

Clinical differentials considered were gluteal abscess, tubercular arthritis, and pyogenic arthritis. Tubercular and pyogenic arthritis were considered as differentials because of the short duration of symptoms which were mainly pain and swelling in the gluteal region.

Radiological examination showed cortical destruction over superior pubic ramus. Right hip joint showed reduced joint space with irregular borders and early sclerosis. Computed tomography scan finding was a large heterogeneous collection of fluid, mixed with bony fragments, around the right hip joint, iliac blade, and also lower psoas and quadratus lumborum muscles, displacing the urinary bladder to the left iliac fossa, and erosion of right iliac blade, acetabulum and head of femur, pubic bone, and ischium (Fig. 1). Computed tomography scan findings were confirmed by magnetic resonance imaging. Radiological differential diagnosis provided tuberculosis or antibioma.

Aspiration of the abscess (swelling) in gluteal region was tried under ultrasound guidance. It did not yield any fluid. Arthrotomy of the right hip was done through the posterior approach. Fluid was aspirated from right hip joint, 2 to $3 \mathrm{~mL}$, serous in nature and sent for culture and sensitivity. It was a sterile tap with no cells. A hard mass around the greater trochanter was found. Incision biopsy was taken and sent for histopathological examination.

Grossly, we received an irregular, nodular, gray-white to gray-brown, firm to hard tissue measuring $3.0 \times 3.0 \times$ $1.5 \mathrm{~cm}$. Cut surface showed gray-white to gray-brown tissue admixed with gelatinous areas (Fig. 2).

Microscopy showed a tumor composed of lobules and islands of malignant cartilaginous tissue separated by fibrous septae. The islands are composed of round to oval chondrocytes exhibiting marked cellular and nuclear atypia, such as hyperchromatism, pleomorphism, binucleate, and multinucleated lacunae. Areas of extensive hyaline and myxoid changes were seen. The tumor was extending up to the adjacent fibromuscular tissue. 

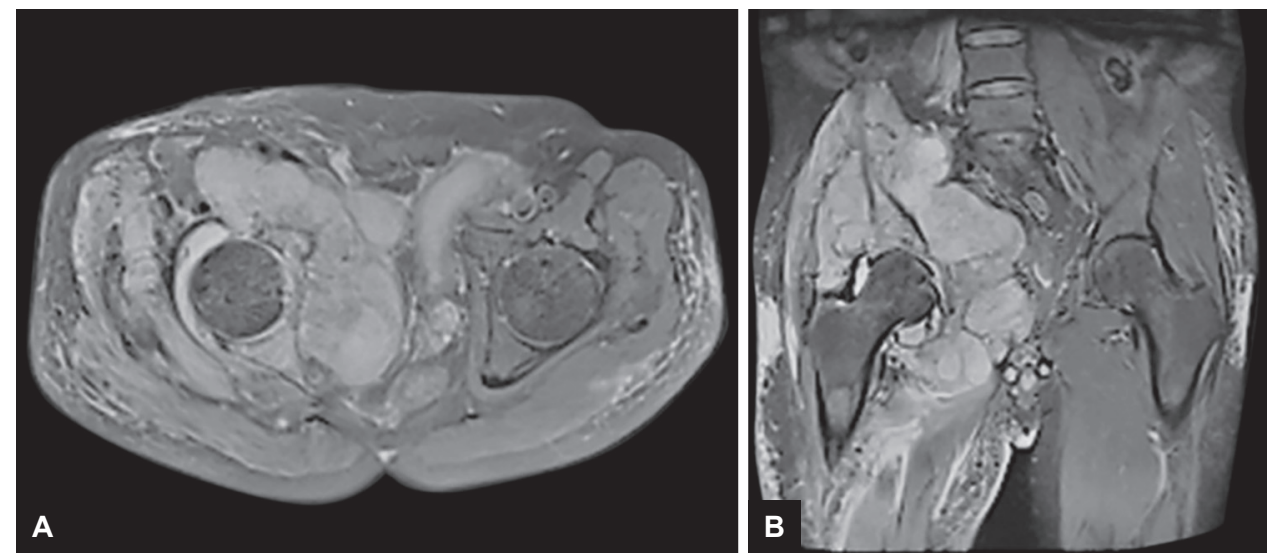

Figs $1 \mathrm{~A}$ and $\mathrm{B}$ : Large irregular heterogeneously enhancing collection involving the superficial and deep muscle planes of right gluteal region extending into anterior and medial compartment of proximal thigh, causing displacement of the surrounding vessels, urinary bladder, rectum, sigmoid colon, and muscles

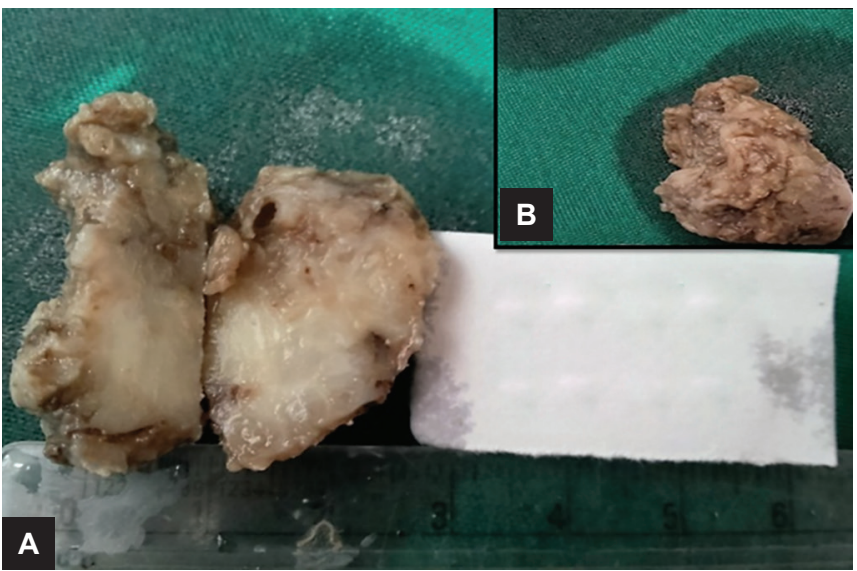

Figs 2A and B: (A) Cut surface showing gray-white to gray-brown tissue admixed with gelatinous areas; and (B) inset shows an irregular, firm, hard gray-white to gray-brown soft tissue bit.

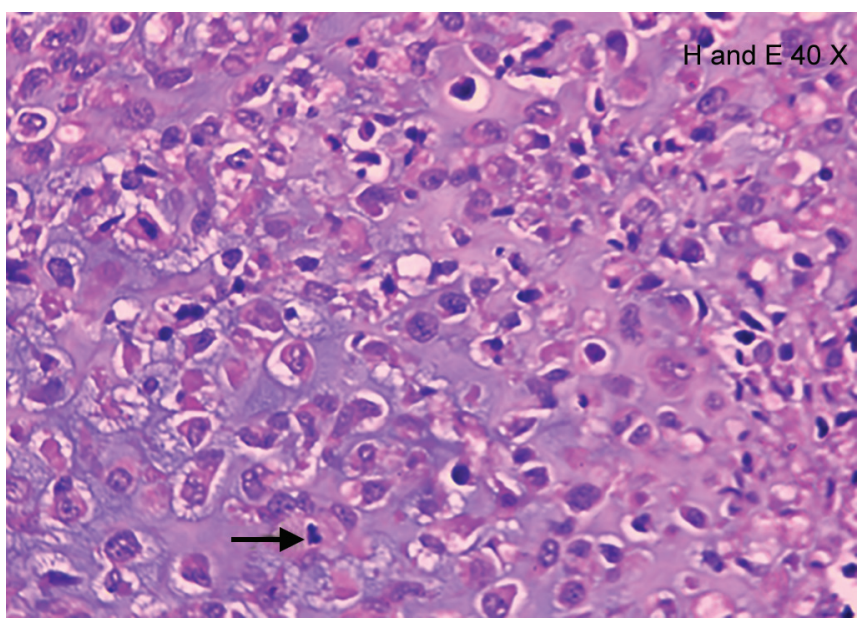

Fig. 4: Islands of atypical chondrocytes with mitotic figures (hematoxylin and eosin, 40x)

Evidence of vascular invasion was also present (Figs 3 to 5). Impression was given as CHS grade II, pelvis.

On diagnosing it as a case of CHS grade II, patient was referred to Kidwai Memorial Institute of Oncology and was lost to follow-up.

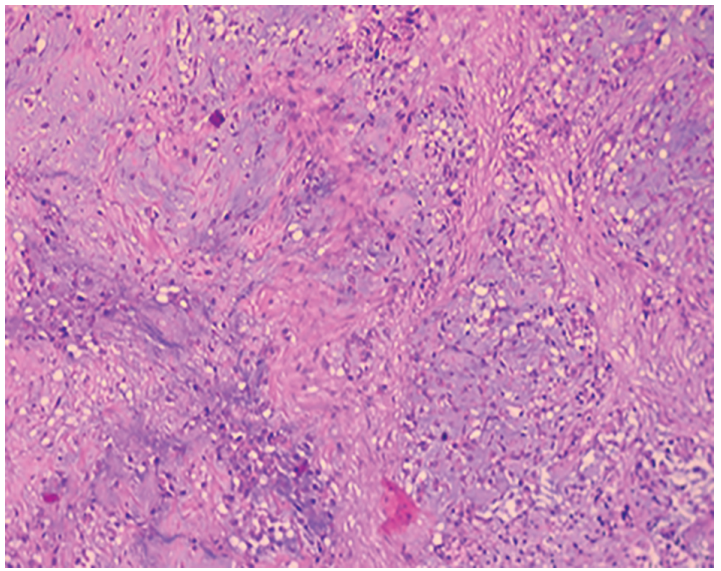

Fig. 3: Tumor composed of islands of malignant cartilaginous tissue (hematoxylin and eosin, 10x)

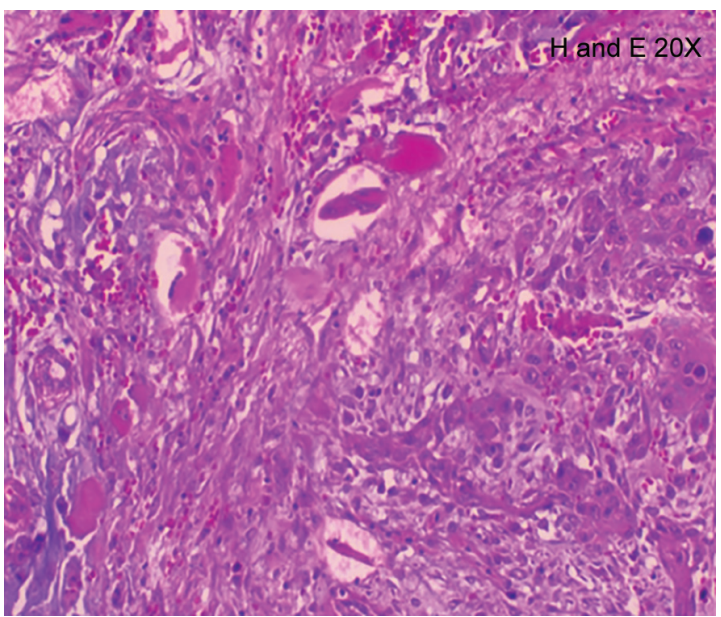

Fig. 5: Adjacent skeletal muscle invasion by tumor cells (hematoxylin and eosin, 20x)

\section{DISCUSSION}

The salient feature of the case under review was the dilemma of diagnosing a CHS, which was confused to be a gluteal abscess. Chondrosarcoma, a malignant tumor of cartilage-forming tissues, is the third most frequent 
malignant neoplasm of bone, occurring less frequently than only myeloma and osteosarcoma. ${ }^{2}$ It is divided into two major categories based on microscopic criteria: Conventional CHS and CHS variants. Each of these categories comprises several distinct types, some defined on microscopic grounds and others based on the location within the affected bone. ${ }^{3}$ There is higher preponderance in axial than appendicular skeleton with the pelvis being most common site accounting for about $40 \%$ of most cases. ${ }^{2}$ Early diagnosis is difficult because patients with pelvic CHS are usually relatively asymptomatic early in the course of the disease and tend to present at a later stage where the tumor has already reached a large size. This is because of the wide space within the pelvic cavity that can accommodate a growing tumor without any compressive symptoms until it is large enough to cause mass effect. The symptoms created by CHS of the pelvis can easily be mistaken for mechanical back pain. Laboratory evaluation including ESR and ALP can also provide additional information. In fact, the ESR can be more sensitive than plane radiographs in the initial diagnosis of spinal cancer as was seen in our case. ${ }^{4}$ The diagnosis of CHS is among the most difficult problems in orthopedic tumor pathology. The histology of CHS was first described by Lichtenstein and Jaffe. It usually contains an abundant amount of hyaline type cartilage, a lobulated growth pattern with round and oval cells in lacunae with enlarged nuclei. Features, such as foci of atypical spindle cells, myxoid degeneration of the matrix, and calcification or ossification of the matrix may be seen. ${ }^{5}$ The absence of osteoid and neoplastic bone in the case presented here ruled out chondroblastic variant of osteosarcoma. Chondrosarcoma is classified histologically from I to III of Evan's classification by nuclear size, differentiation, and nuclear pleomorphism. Chondrosarcoma is one of the few tumors in which microscopic grading has a significant prognostic value. Grading is based on cellularity of the lesion and the nuclear changes of the chondrocytes. Grade I CHSs show increased cellularity compared with an enchondroma. The chondrocytes are enlarged and more irregular. Grade II CHSs show even more cellularity than grade I CHSs, and the nuclear changes are more pronounced. Grade III CHSs show marked cellularity, marked pleomorphism of the nuclei, and some spindling at the periphery of the lobules. ${ }^{6}$ Wide surgical tumor excision with adequate margins appears to be the procedure of choice in CHS treatment, since this is the most effective way of reducing tumor recurrence rate. ${ }^{7}$ The 5 -year survival is $89 \%$ for patients with grade I, the combined group of patients with grades II and III have a 5 -year survival of $53 \%{ }^{1}$

\section{CONCLUSION}

Chondrosarcomas are malignant tumors with a predilection for the pelvis. Their presentation may simulate mechanical low back pain and mass lesions like infections and abscesses. The clinician should be aware of such a possibility in the differential diagnosis of patients presenting with the same to avoid making a misdiagnosis. Because at times rare lesions are readily diagnosed as a result of their rarity, the common ones go unnoticed as they are less sought after.

\section{REFERENCES}

1. Fletcher C, Krishnan Unni K, Mertens F. Chapter 10: cartilage tumor. In: Pathology and genetics of tumors of soft tissue and bone. Lyon: IARC Press; 2002. pp. 247-249.

2. Fletcher CD, Inwards $C Y$, Oliveira AM. Chapter 25: tumors of the osteoarticular system. In: Fletcher CD, editor. Diagnostic histopathology of tumors. Philadelphia (PA): Saunders/ Elsevier; 2013. pp. 1884-1890.

3. Rosai J, Ackerman L. Chapter 24: bone and joints. In: Rosai and Ackerman's surgical pathology. Edinburgh: Mosby; 2011. pp. 2038-2042.

4. Hains F, David Cassidy J, Dust W. Pelvic chondrosarcoma presenting as mechanical back pain: two case reports. J Can Chiropr Assoc 1993 Mar;37(1):15-21.

5. Kundu S, Pal M, Paul RR. Clinicopathologic correlation of chondrosarcoma of mandible with a case report. Contemp Clin Dent 2011 Oct;2(4):390-393.

6. Sharma M, Madan M, Manjari M, Kaur H. Pituitary chondrosarcoma presenting as a sellar and suprasellar mass with parasellar extension: an unusual presentation. Iran J Pathol 2016 Spring;11(2):161-166.

7. Mahajan AM, Ganvir SM, Hazarey VK, Mahajan MC. Chondrosarcoma of the maxilla: a case report and review of literature. J Oral Maxillofac Pathol 2013 Oct;17(2):269-273. 